\title{
Influence of the Grain Size on Polaron Transport in Nanostructured $\mathrm{La}_{2 / 3} \mathrm{Ca}_{1 / 3} \mathrm{MnO}_{3}$ Evidenced by EPR
}

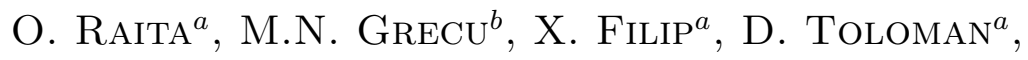 \\ L.M. Giurgiu ${ }^{a, *}$, S. IdziaK ${ }^{c}$ And S.K. HofFmanN ${ }^{c}$ \\ ${ }^{a}$ National Institute for Research and Development of Isotopic and Molecular \\ Technologies, P.O. Box 700, 400293, Cluj-Napoca, Romania \\ ${ }^{b}$ National Institute for Material Physics, 76900 Bucharest-Magurele, Romania \\ ${ }^{c}$ Institute of Molecular Physics, Polish Academy of Sciences \\ 60-179 Poznań, Poland

\begin{abstract}
We report an electron paramagnetic resonance investigation of the grain size effects on the polaron transport in the paramagnetic regime of nanosized $\mathrm{La}_{2 / 3} \mathrm{Ca}_{1 / 3} \mathrm{MnO}_{3}$ manganites. The temperature dependences of the EPR integral intensity were analyzed in terms of spin-spin exchange interaction and small polaron hopping scenarios. The polaron activation energy decreases with the reduction of the grain size. A discussion is given concerning the factors which could explain the observed change.
\end{abstract}

PACS numbers: 75.50.Tt, 73.63.Bd, 76.50.+g

\section{Introduction}

The perovskite manganites $\mathrm{La}_{1-x} \mathrm{Ca}_{x} \mathrm{MnO}_{3-\delta}$ (LCMO) have been intensively studied due to their colossal magnetoresistive (CMR) properties. The basic features involve a mixture of the so-called double exchange (DE) interaction $\mathrm{Mn}^{3+}-\mathrm{O}-\mathrm{Mn}^{4+}[1]$ and a strong spin-lattice interaction [2] which determines the presence of the Jahn-Teller (JT) polarons in the paramagnetic (PM) regime [3]. An analysis of LCMO and $\mathrm{LaMnO}_{3-\delta}$ series has shown a strong increase in the polaron activation energy $E_{\mathrm{a}}$, as $\mathrm{Mn}^{3+}$ content increases [4]. It was argued that the polaron binding energy was proportional to the $\mathrm{Mn}-\mathrm{O}$ distortion and the $\mathrm{Mn}^{3+}$ content [5].

${ }^{*}$ corresponding author; e-mail: giurgiu@s3.itim-cj.ro 
From the viewpoint of future technological applications, CMR manganites with nanometer-scale dimensions will be required. These nanosized materials could exhibit enhanced electronic and magnetic properties compared with their conventional microscaled counterparts [6].

Electron paramagnetic resonance (EPR) spectroscopy could give useful information about the dynamics of spins over a wide temperature range. A number of EPR studies have been performed in ceramic [7, 8] as well as nanometric $[9,10]$ CMR manganites in order to clarify the dynamic magnetic correlations on a microscopic level. In our previous publications we presented systematic EPR investigations of the spin dynamics in microscaled $\mathrm{La}_{2 / 3} \mathrm{Ca}_{1 / 3} \mathrm{Mn}_{1-x} \mathrm{Me}_{x} \mathrm{O}_{3}(\mathrm{Me}=$ $\mathrm{Al}, \mathrm{In} ; x \leq 0.05)[11,12]$ and nanosized $\mathrm{La}_{2 / 3} \mathrm{Ca}_{1 / 3} \mathrm{MnO}_{3-\delta}$ manganites [13]. The experimental data have been analyzed in terms of the bottlenecked spin relaxation and small polaron hopping scenarios [14].

In the present work we investigated by EPR the influence of the grain size reduction on $E_{\mathrm{a}}$ in nanostructured LCMO. Here, a different approach based on the spin-spin exchange interaction scenario was used to analyze in the PM regime the temperature dependence of the EPR integral intensity $[7,15]$.

\section{Sample characterization}

Granular nanosized $\mathrm{La}_{2 / 3} \mathrm{Ca}_{1 / 3} \mathrm{MnO}_{3-\delta}$ manganites were prepared by the sol-gel technique. Two samples with different grain size were obtained by annealing at the temperatures $T_{\mathrm{A}}=973$ and $1373 \mathrm{~K}$, respectively.

Magnetic, scanning electron microscopy (SEM), and redox titration investigations were carried out in order to evaluate the magnetic transition temperature $T_{\mathrm{c}}$, the mean grain size $D$, and the $\mathrm{Mn}^{4+}$ content, respectively. The estimated values are shown in Table I and compared with the corresponding ones for a microsized (ceramic) sample. As it is observed, the grain size and $T_{\mathrm{c}}$ decrease as the annealing temperature decreases.

TABLE I

Annealing temperature $T_{\mathrm{A}}$, mean grain size $D$, percentage of $\mathrm{Mn}^{4+}$, and transition temperature $T_{\mathrm{c}}$ for $\mathrm{La}_{2 / 3} \mathrm{Ca}_{1 / 3} \mathrm{MnO}_{3}$ samples.

\begin{tabular}{c|c|c|c}
\hline \hline $\mathrm{La}_{2 / 3} \mathrm{Ca}_{1 / 3} \mathrm{MnO}_{3}$ & $D[\mathrm{~nm}]$ & $\mathrm{Mn}^{4+}[\%]$ & $T_{\mathrm{c}}[\mathrm{K}]$ \\
\hline ceramic, $T_{\mathrm{A}}=1723 \mathrm{~K}$ & $>1000$ & 30 & 263 \\
nanosized, $T_{\mathrm{A}}=1373 \mathrm{~K}$ & 150 & 32 & 245 \\
nanosized, $T_{\mathrm{A}}=973 \mathrm{~K}$ & 90 & 40 & 201
\end{tabular}

The high-temperature annealed nanosized sample is nearly stoichiometric but the lower-temperature one presents $\mathrm{Mn}^{4+}$ in excess. This could mean that the samples have different oxygen content. 


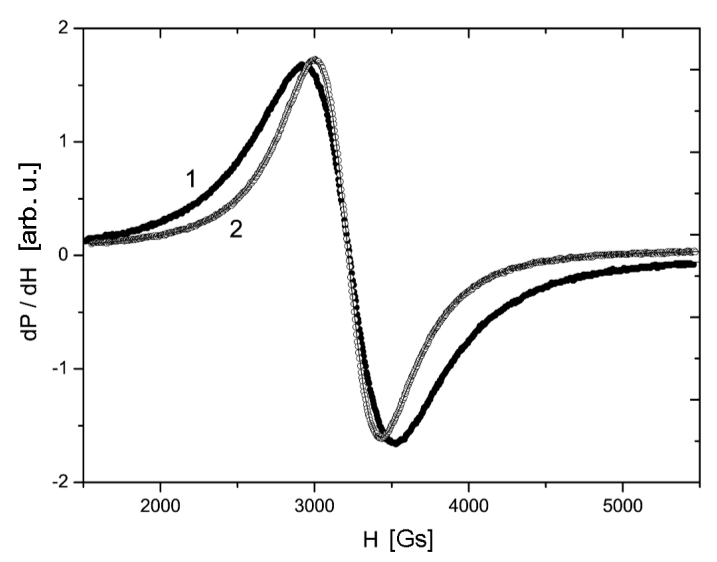

Fig. 1. EPR spectra at $T=300 \mathrm{~K}$ for $\mathrm{La}_{2 / 3} \mathrm{Ca}_{1 / 3} \mathrm{MnO}_{3-\delta}$ with different grain sizes: $1-D=90 \mathrm{~nm}, 2-D=150 \mathrm{~nm}$. The solid lines represent the fits with the Lorentzian line shape.

X-band EPR investigations were carried out in the temperature range 100$550 \mathrm{~K}$. In the PM regime the EPR spectrum of nanosized LCMO samples consists of a single line with $g \approx 2$ similar to that observed for ceramic samples [12]. The line shape was found to be Lorentzian over the investigated temperature range and independent of the grain size (Fig. 1).

\section{EPR integral intensity in PM regime}

According to the spin-spin exchange interaction scenario, in the PM regime the EPR integral intensity, $I_{\mathrm{EPR}}$, is determined by all of $\mathrm{Mn}^{3+}$ and $\mathrm{Mn}^{4+}$ ions present in the lattice and the main variation of the line width is given by [7]:

$$
\Delta H(T) \propto 1 / T \chi_{\mathrm{dc}},
$$

where $\chi_{\mathrm{dc}}$ is the static susceptibility.

In the systems with hopping conductivity, $\Delta H(T)$ was found to be proportional to the conductivity: $\Delta H(T) \propto \sigma(T)[16]$. On the other hand, the small polaron hopping conductivity in the PM regime of LCMO manganites has the following temperature dependence [17]:

$$
\sigma(T) \propto(1 / T) \exp \left(-E_{\mathrm{a}} / k T\right) .
$$

Finally, taking into account that $I_{\mathrm{EPR}} \propto \chi_{\mathrm{dc}}$ one gets [15]:

$$
I_{\mathrm{EPR}}(T)=I_{0} \exp \left(E_{\mathrm{a}} / k T\right) .
$$

\section{Results and discussion}

In the following, the effects of the grain size reduction on the polaron activation energy in PM regime of nanometric $\mathrm{La}_{2 / 3} \mathrm{Ca}_{1 / 3} \mathrm{MnO}_{3-\delta}$ are presented and discussed in terms of LCMO nanoparticle model [6]. 
We can get an estimate of $E_{\mathrm{a}}$ by fitting Eq. (3) to the $I_{\mathrm{EPR}}=f(T)$ data (Fig. 2). In Fig. 2 the solid lines represent the best fit to Eq. (3) in the temperature range $1.3 T_{\mathrm{c}} \leq T \leq 500 \mathrm{~K}$. The results can be found in Table II and compared with the evaluated $E_{\mathrm{a}}$ value by means of Eq. (3) for a ceramic sample with micrometer size grains. These values are larger than the corresponding ones obtained from the analysis of the line width temperature dependence [13].

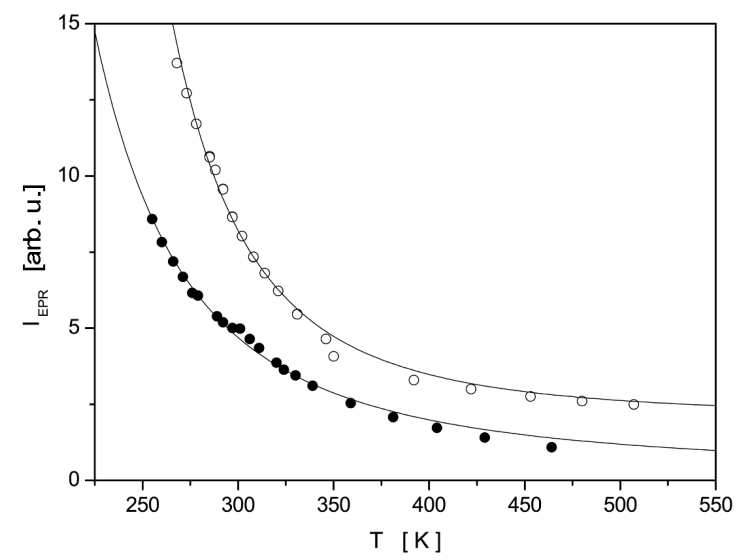

Fig. 2. Comparison of the temperature dependences of $I_{\mathrm{EPR}}(T)$ in the PM regime of $\mathrm{La}_{2 / 3} \mathrm{Ca}_{1 / 3} \mathrm{MnO}_{3-\delta}$ samples with mean grain sizes of 150 (open circles) and $90 \mathrm{~nm}$ (black circles). The solid lines are the best fit to Eq. (3).

\section{TABLE II}

Polaron activation energy $E_{\mathrm{a}}$ in the paramagnetic regime of nanosized $\mathrm{La}_{2 / 3} \mathrm{Ca}_{1 / 3} \mathrm{MnO}_{3-\delta}$ manganites.

\begin{tabular}{c|c}
\hline \hline $\mathrm{La}_{2 / 3} \mathrm{Ca}_{1 / 3} \mathrm{MnO}_{3-\delta}$ & $E_{\mathrm{a}}[\mathrm{meV}]$ \\
\hline ceramic, $D>1000 \mathrm{~nm}$ & 186 \\
nanosized, $D=150 \mathrm{~nm}$ & 148 \\
nanosized, $D=90 \mathrm{~nm}$ & 89
\end{tabular}

As one can see from Table II, the overall result of the grain size reduction is the decrease in $E_{\mathrm{a}}$. This behaviour might be explained in terms of the so-called inner core-outer shell scenario [6].

In accordance with this, each LCMO nanoparticle is composed of two different parts: an inner ferromagnetically ordered core with the same properties as the bulk compound and an outer shell (surface layer). The magnetic configuration in the grain surface is more chaotic than in the core due to presence of the oxygen 
vacancies, superficial stress, dangling bonds, etc. The decrease in $E_{\text {a }}$ with decreasing grain size could be attributed to two opposite contributions arising from the inner core and surface layer of the nanometric particles.

The inner core contribution results from the different $\mathrm{Mn}^{4+}$ content in our nanosized samples. From Table II one can see that $E_{\mathrm{a}}$ decreases with decreasing grain size, i.e. the increasing $\mathrm{Mn}^{4+}$ content or similarly, decreasing $\mathrm{Mn}^{3+}$ content. Therefore, based on the paper [4] one would expect a decrease in $E_{\text {a }}$ with the increase in the $\mathrm{Mn}^{4+}$ content.

It is well known that the influence of the outer shell increases as the particle size decreases. Moreover, a strong influence of the magnetically disordered surface on the conduction mechanism in manganites is expected, since inside the surface the spins of $\mathrm{Mn}^{3+}$ and $\mathrm{Mn}^{4+}$ pairs may not be parallel [18]. As a result, the polaron hopping between $\mathrm{Mn}^{3+}$ and $\mathrm{Mn}^{4+}$ ions is difficult. The presence of the disordered outer shell can play the part of the higher potential barrier for the polarons to hop over and one could expect it to contribute to higher $E_{\mathrm{a}}$ [17].

As the disorder in outer shell is larger in the smaller grains, an increase in $E_{\mathrm{a}}$ with decreasing grain size could be expected.

Therefore, the total result of decreasing $E_{\mathrm{a}}$ as the grain size diminished could arise from two opposite effects: the first effect represents the decrease in $E_{\text {a }}$ with increasing $\mathrm{Mn}^{4+}$ content and the second one corresponds to the increase in $E_{\mathrm{a}}$ due to the increased disorder in the outer shell of the smaller grains. Our investigations point out to dominant core-shell contribution to $E_{\mathrm{a}}$ as shown by the results presented in Table II.

\section{Conclusions}

Based on the spin-spin exchange interaction and small polaron hopping scenarios, the polaron activation energy $E_{\mathrm{a}}$ was evaluated from the temperature dependence analysis of the EPR integral intensity. The observed decrease in $E_{\mathrm{a}}$ with the reduction of the grain size is a global result which could be accounted for by both the inner core contribution and the influence of outer shell. $E_{\text {a }}$ decreases with the increase in $\mathrm{Mn}^{4+}$ content, while an increased disorder in the surface layer of the smaller grains results in the increase in $E_{\mathrm{a}}$. Since the overall effect is the decrease in $E_{\mathrm{a}}$, the inner core contribution to $E_{\mathrm{a}}$ is dominant.

\section{Acknowledgments}

L.M. Giurgiu acknowledges fruitful discussions with M. Mehring and G. Papavassiliou. We thank for the financial support from MECT, MATNANTECH Romanian National Research Program no. 268(409). 


\section{References}

[1] C. Zener, Phys. Rev. 82, 403 (1951).

[2] H. Röder, J. Zhang, A. Bishop, Phys. Rev. Lett. 76, 1356 (1996).

[3] M. Jaime, M.B. Salamon, M. Rubinstein, R.E. Treece, J.S. Horwitz, D.B. Chrisey, Phys. Rev. B 54, 11914 (1996).

[4] J.M. deTeresa, K. Dörr, K.H. Müller, L. Schultz, R.I. Chakalova, Phys. Rev. B 58, R5928 (1998).

[5] C.H. Booth, F. Bridges, G.H. Kwei, J.M. Lawrence, A.L. Cornelius, J.J. Neumeier, Phys. Rev. Lett. 80, 853 (1998).

[6] M.A. Lopez-Quintela, L.E. Hueso, J. Rivas, F. Rivadulla, Nanotechnology 14, 212 (2003).

[7] D.L. Huber, G. Alejandro, A. Caneiro, M.T. Causa, F. Prado, M. Tovar, S.B. Osero, Phys. Rev. B 60, 12155 (1999).

[8] A.I. Shames, E. Rozenberg, G. Gorodetsky, Ya.M. Mukovskii, Phys. Rev. B 68, 174402 (2003).

[9] L. Malavasi, M.C. Mozzati, S. Polizzi, C.B. Azzoni, G. Flor, Chem. Mater. 15, 5036 (2003).

[10] S. Das, P. Chowdhury, T.K.G. Rao, D. Das, D. Bahadur, Solid State Commun. 121, 691 (2002).

[11] L.V. Giurgiu, M.N. Grecu, X. Filip, O. Raita, A. Darabont, D. Gavre, J. Blasco, Appl. Magn. Reson. 24, 351 (2003).

[12] O. Raita, M.N. Grecu, X. Filip, A. Darabont, D. Gavre, J. Blasco, L.V. Giurgiu, J. Optoelectron. Adv. Mater. 5, 289 (2003).

[13] L.M. Giurgiu, M.N. Grecu, A. Darabont, O. Raita, X. Filip, O. Pana, D. Toloman, Appl. Magn. Reson. 27, 139 (2004).

[14] A. Shengelaya, G.M. Zhao, H. Keller, K.A. Müller, B.I. Kochelaev, Phys. Rev. B 61, 5888 (2000).

[15] D.S. Yang, A.N. Ulyanov, M.H. Phan, I. Kim, B.K. Ahn, J.R. Rhee, J.S. Kim, C. Nguyen, S.C. Yu, Physica B 327, 183 (2003).

[16] O. Chauvet, T. Stoto, L. Zuppiroli, Phys. Rev. B 49, 5876 (1994).

[17] D.C. Worledge, G.F. Snyder, M.R. Beasley, T.H. Geballe, J. Appl. Phys. 80, 5158 (1996).

[18] H.L. Ju, J. Gopalakrishnan, J.L. Peng, Q. Li, G.C. Xiong, T. Venkatesan, R.L. Green, Phys. Rev. B 51, 6143 (1995). 